\title{
ACESSO VASCULAR PARA HEMODIÁLISE: AVALIAÇÃO DO TIPO E LOCAL ANATÔMICO EM 23 UNIDADES DE DIÁLISE DISTRIBUÍDAS EM SETE ESTADOS BRASILEIROS.
}

\author{
HEMODIALYSIS VASCULAR ACCESS: EVALUATION OF TYPE AND LOCAL OF \\ VASCULAR ACCESS USED IN 23 DIALYSIS CENTERS IN SEVEN BRAZILIAN \\ STATES.
}

\author{
Fábio Linardi ${ }^{1}$ \\ Felipe de Francisco Linardi ${ }^{2}$ \\ José Luís Bevilacqua ${ }^{3}$ \\ José Francisco Moron Morad ${ }^{4}$ \\ José Augusto Costa ${ }^{5}$ \\ Fausto Miranda Júnior, TCBC-SP'
}

\begin{abstract}
RESUMO: Objetivo: Demonstrar os acessos vasculares para hemodiálise mais utilizados em 23 unidades de hemodiálise, distribuídas em sete estados brasileiros. Método: Entre outubro de 1999 a agosto de 2000, foram avaliados 2559 pacientes em 23 unidades de hemodiálise distribuídas em 23 estados brasileiros onde foi observado: A - A freqüência da utilização do acesso vascular, se externo através de cateteres ou se interno através de fístula arteriovenosa (FAV). B - Os tipos de cateteres, se de curta permanência ou de longa permanência, assim como os locais anatômicos utilizados para sua inserção. C-Os tipos de FAV, se direta ou com interposição de algum tipo de prótese e os locais anatômicos onde foram construídas, se distais ou proximais. $\mathrm{D}$ - $\mathrm{O}$ custo financeiro com os acessos vasculares. Resultados: Constatou-se que 93,4\% dos pacientes tinham um acesso vascular através de FAV e 6,6\% através de cateter. As FAV diretas distais foram as mais utilizadas em 74,8\% dos pacientes; as FAV diretas proximais foram construídas em $21,7 \%$ das vezes; as FAV com politetrafluoretileno expandido (PTFE) 3,2\% da totalidade; a veia safena foi utilizada em $0,1 \%$ e as FAV consideradas como outras em $0,2 \%$. Os cateteres de longa permanência foram utilizados em $8,7 \%$ da totalidade dos cateteres e os de curta permanência em $91,3 \%$. Como via de acesso a veia jugular foi utilizada em $42,4 \%$, a veia subclávia em $42,4 \%$ e a veia femoral em $6,5 \%$. O custo financeiro com acesso para hemodiálise foi de $1 \%$ do custo total das unidades. Conclusões: O acesso vascular no Brasil tem características próprias. O acesso mais utilizado é a FAV distal e o uso do PTFE é baixo.
\end{abstract}

Descritores: Hemodiálise; Fístula arteriovenosa; Acesso vascular; Cateter; Insuficiência renal crônica

\section{INTRODUÇÃO}

O tratamento dos pacientes portadores de insuficiência renal crônica terminal está baseado nos programas de hemodiálise, diálise peritoneal e transplante renal, sendo a hemodiálise a terapêutica de maior alcance na atualidade. Os dados epidemiológicos brasileiros são raros e estão baseados

1. Assistente Doutor do Departamento de Cirurgia da Faculdade de Ciências Médicas do CCMB PUC-SP

2. Acadêmico da Faculdade de Medicina de Bragança Paulista - USF

3. Nefrologista do Serviço de Nefrologia do Hospital Evangélico de Sorocaba

4. Assistente Mestre do Departamento de Morfologia e Patologia da Faculdade de Ciências Médicas do CCMB - PUC-SP

5. Prof. Titular do Departamento da Faculdade de Ciências Médicas do CCMB - PUC-SP

6. Prof. Adjunto Livre Docente da Faculdade da Escola Paulista de Medicina - UNIFESP

Recebido em: 19/04/2002

Aceito para publicação em 08/10/2002

Trabalho realizado na Faculdade de Ciências Médicas do CCMB - PUC-SP, na Escola Paulista de Medicina - UNIFESP e no Renal Therapy Service - RTS. 
nos dados do Data SUS ${ }^{1}$ e em trabalho publicado por $\mathrm{Sesso}^{2}$ em 2000. Em ambos os estudos, estima-se que aproximadamente 40000 pacientes estejam em programa de hemodiálise no Brasil, com uma prevalência de 268 e 287 por milhão da população (pmp), respectivamente. Em relação aos acessos vasculares, a única referência é encontrada no trabalho de $\mathrm{Sesso}^{2}$ que demonstra $8 \%$ de utilização de cateteres e $2 \%$ de utilização de próteses.

A literatura nacional sobre acesso vascular é pobre e, a grande maioria dos trabalhos são encontrados na literatura internacional que, apresenta resultados diferentes da realidade nacional.

A literatura americana atual, apresenta alto índice do uso de PTFE naquele país que chega atingir cifras de $85 \%$ do total dos acessos, elevando o custo financeiro a mais de um bilhão de dólares ao ano com o acesso vascular e foi responsável por $25 \%$ do total das hospitalizações dos pacientes portadores de insuficiência renal crônica terminal. ${ }^{3}$

Fica claro que um estudo epidemiológico sobre o acesso vascular para a nossa realidade é de fundamental importância tanto para os cirurgiões, os nefrologistas e enfermeiros envolvidos com os pacientes renais crônicos. A finalidade deste trabalho é demostrar quais os acessos vasculares para hemodiálise mais utilizados em nosso meio.

\section{MÉTODO}

A casuística é composta de 2559 pacientes portadores de insuficiência renal crônica terminal em programa de hemodiálise. O estudo teve início em outubro de 1999 e se findou em agosto de 2000. Durante esse período foram visitadas 23 unidades de hemodiálise localizadas em sete estados brasileiros que, recebem orientação administrativa da Renal Therapy Service (RTS).

A RTS, é uma empresa que oferece orientação administrativa a unidades de hemodiálise e, possui um conselho médico denominado de Medical Advisor Boarding (MAB), que por sua vez auxilia todas as unidades, orientando, quando necessário, ou por solicitação, nos aspectos clínicos que envolvem uma unidade de hemodiálise.

A partir de setembro de 1998, o autor foi convidado para realizar consultoria em acesso vascular para hemodiálise e visitou as 23 unidades nas quais a empresa atuava. Durante as visitas, o autor examinou pessoalmente 2559 pacientes e coletou os dados referentes aos tipos de acesso vascular para hemodiálise através do qual o paciente estava seno dialisado no momento do exame. Os dados referentes aos custos financeiros, foram obtidos junto ao departamento financeiro da RTS, através de planilhas de custo da empresa durante os meses de janeiro a outubro de 2000, nas quais foram considerados o custo mensal total de uma unidade, o custo mensal total com o acesso, considerando, taxas hospitalares, material, medicamento e honorários médicos e a relação em percentual entre esses dois custos.

Os acessos vasculares foram classificados em três grupos:

A - Cateter de curta permanência: aqueles que não possuem "cuff" de Dacron $^{\mathrm{R}}$ e são inseridos sem contra abertura da pele através de punção direta do vaso. São denominados de cateter de Shiley e utilizados por tempo curto com a finalidade de se prover um acesso vascular imediato em pacientes com insuficiência renal em urgência dialítica ou durante o período de maturação de um acesso definitivo. O local anatômico utilizado para a inserção do cateter de curta permanência, veia jugular, ou veia subclávia ou veia femoral, também foi utilizado para essa classificação.

B - Cateter de longa permanência: aqueles que possuem "cuff" de Dacron ${ }^{\mathrm{R}}$ e são inseridos por contra abertura da pele. Na prática diária são conhecidos como "permcath" (PC). O local anatômico para a inserção do cateter de longa permanência não foi considerado para análise, visto que a veia jugular interna direita é a via de eleição para a inserção desse tipo de cateter.

\section{C - Fístula arteriovenosa}

As fistulas arteriovenosas foram classificadas

em:

1 - Fístula arteriovenosa direta: aquelas realizadas através de ato cirúrgico em que ocorreu pelo menos uma anastomose entre uma artéria e uma veia, próprias do paciente. As fistulas arteriovenosas diretas foram subdivididas em dois grupos:

a) Distais: aquelas realizadas abaixo da prega do cotovelo, com apenas uma anastomose vascular e utilizando somente artérias e veias da região.

b) Proximais: aquelas realizadas na região da prega do cotovelo, com apenas uma anastomose vascular e utilizando somente artérias e veias da região

2 - Fístula arteriovenosa com interposição de segmento de politetrafluoretileno expandido (PTFE): 
realizadas através de ato cirúrgico e interposto segmento de PTFE entre uma artéria e uma veia independente do local anatômico.

3 - Fístula arteriovenosa com interposição de segmento de veia safena autógena: realizadas através de ato cirúrgico, no qual foi interposto segmento de veia safena autógena entre uma artéria e uma veia independente do local anatômico.

4 - Outras: as FAV realizadas no membro inferior utilizando artéria e veia da região, seja na perna ou coxa.

Para melhor elaboração dos dados obtidos, esses foram agrupados em três situações:

1 - Número total de pacientes examinados: o resultado foi obtido através do total dos pacientes examinados.

2 - Por estados da União: foram agrupados os dados referentes a soma das unidades localizadas em cada estado da União.

3 - Por situação geográfica: foram agrupados os resultados referentes a soma das unidades localizadas nas regiões geográficas do Brasil: Sul, Sudeste e Nordeste.

4 - Custos financeiros com acesso vascular: foram considerados o custo total na construção de um acesso vascular: taxas hospitalares, materiais e medicamentos e honorários médicos e a relação em percentual com o custo mensal total da unidade.

\section{RESULTADOS}

1 - Número total de pacientes:

Da totalidade dos pacientes estudados (2559), foi observado que $2389(93,4 \%)$ tinham acesso vascular para hemodiálise através de FAV e 170 (6,6\%) estavam sendo submetidos a sessões de hemodiálise por acesso vascular temporário através de cateter. Os resultados estão representados na tabela 1.

\section{2 - Estados da União}

Nos sete estados da União estudados as unidades de hemodiálise estavam assim distribuídas: Rio Grande do Sul uma unidade, Paraná uma unidade, São Paulo sete unidades, Rio de Janeiro cinco unidades, Minas Gerais seis unidades, Bahia duas unidades e Ceará uma unidade. Os resultados estão representados nas tabelas 2 e 3.

3 - Situação geográfica

Nas três regiões brasileiras visitadas havia duas unidades na região sul, dezoito na sudeste e três na nordeste. Os resultados estão representados nas tabelas 4 e 5 .

4 - Custos financeiros dos acessos vasculares

$\mathrm{O}$ custo financeiro com acesso vascular para hemodiálise nas 23 unidades da RTS, representou 1,0\% do custo total dessas unidades. Entre as 23 unidades, houve uma variação de custo de $0,3 \%$ a $2,6 \%$. Em relação aos estados e a situação geográficas, os custos estão representados nas tabelas 3 e 5 respectivamente.

Tabela 1 - Distribuição dos pacientes submetidos a acesso vascular para hemodiálise segundo o tipo de acesso e local anatômico.

\begin{tabular}{rcr}
\hline Variáveis & Freqüência & Porcentagem \\
FAV & 2389 & $93,4 \%$ \\
Cateter & 170 & $6,6 \%$ \\
Total & 2559 & $100,0 \%$ \\
Fístula Arteriovenosa & & \\
Distal & 1787 & $74,8 \%$ \\
Proximal & 517 & $21,7 \%$ \\
Ptfe & 77 & $3,2 \%$ \\
Safena & 3 & $0,1 \%$ \\
Outras & 5 & $0,2 \%$ \\
Total & 2389 & $100,0 \%$ \\
Cateter & & \\
Longa Permanência & 15 & $8,7 \%$ \\
Curta Permanência & 72 & $42,4 \%$ \\
Jugular & 72 & $42,4 \%$ \\
Subclávia & 11 & $6,5 \%$ \\
Femoral & 170 & $100,0 \%$ \\
\hline
\end{tabular}


Tabela 2 - Distribuição dos pacientes submetidos a acesso vascular para hemodiálise, nos estados segundo o tipo de acesso e local anatômico em números absolutos.

\begin{tabular}{rrrrrrrrr}
\hline Variáveis/Estados & RS & PR & SP & RJ & MG & BA & CE & TOTAL \\
\hline FAV & 103 & 125 & 638 & 506 & 609 & 274 & 134 & 2389 \\
Cateter & 1 & 6 & 36 & 18 & 58 & 35 & 16 & 170 \\
Total & 104 & 131 & 674 & 524 & 667 & 309 & 150 & 2559 \\
& & & & & & & & \\
Fístula Arteriovenosa & & & & & & & & \\
Distal & 51 & 103 & 463 & 358 & 459 & 231 & 122 & 1787 \\
Proximal & 47 & 20 & 146 & 132 & 122 & 41 & 9 & 517 \\
Ptfe & 5 & 1 & 28 & 13 & 26 & 2 & 2 & 77 \\
Safena & 0 & 1 & 1 & 0 & 1 & 0 & 0 & 3 \\
Outras & 0 & 0 & 0 & 3 & 1 & 0 & 1 & 5 \\
Cateter & & & & & & & & \\
Longa Permanência & 0 & 0 & 9 & 0 & 4 & 1 & 1 & 15 \\
Curta Permanência & & & & & & & & \\
Jugular & 0 & 1 & 10 & 6 & 31 & 13 & 11 & 72 \\
Subclávia & 1 & 5 & 17 & 12 & 20 & 17 & 0 & 72 \\
Femoral & 0 & 0 & 0 & 0 & 3 & 4 & 4 & 11 \\
\hline
\end{tabular}

Tabela 3 - Distribuição dos pacientes submetidos a acesso vascular para hemodiálise, nos estados segundo o tipo de acesso, local anatômico e custo financeiro, em porcentagem.

\begin{tabular}{rrrrrrrr}
\hline Variáveis/Estados & \multicolumn{1}{c}{ RS } & \multicolumn{1}{c}{ PR } & \multicolumn{1}{c}{ SP } & \multicolumn{1}{c}{ RJ } & \multicolumn{1}{c}{ MG } & \multicolumn{1}{c}{ BA } & CE \\
FAV & $99,00 \%$ & $95,50 \%$ & $94,60 \%$ & $96,50 \%$ & $91,30 \%$ & $88,70 \%$ & $89,30 \%$ \\
Cateter & $1,00 \%$ & $4,50 \%$ & $5,40 \%$ & $3,50 \%$ & $8,70 \%$ & $11,30 \%$ & $10,70 \%$ \\
& & & & & & & \\
Fístula Arteriovenosa & & & & & & & \\
Distal & $49,0 \%$ & $82,4 \%$ & $72,6 \%$ & $71,0 \%$ & $75,3 \%$ & $84,2 \%$ & $91,0 \%$ \\
Proximal & $46,0 \%$ & $16,0 \%$ & $22,9 \%$ & $26,0 \%$ & $20,0 \%$ & $15,0 \%$ & $6,7 \%$ \\
Ptfe & $5,0 \%$ & $0,8 \%$ & $4,4 \%$ & $2,5 \%$ & $4,3 \%$ & $0,8 \%$ & $1,5 \%$ \\
Safena & $0,0 \%$ & $0,8 \%$ & $0,1 \%$ & $0,0 \%$ & $0,2 \%$ & $0,0 \%$ & $0,0 \%$ \\
Outras & $0,0 \%$ & $0,0 \%$ & $0,0 \%$ & $0,5 \%$ & $0,2 \%$ & $0,0 \%$ & $0,8 \%$ \\
& & & & & & & \\
Cateter & & & & & & & \\
Longa Permanência & $0,0 \%$ & $0,0 \%$ & $25,0 \%$ & $0,0 \%$ & $6,9 \%$ & $2,9 \%$ & $6,3 \%$ \\
Curta Permanência & & & & & & & \\
Jugular & $0,0 \%$ & $16,7 \%$ & $27,8 \%$ & $33,3 \%$ & $53,5 \%$ & $37,1 \%$ & $68,7 \%$ \\
Subclávia & $100,0 \%$ & $83,3 \%$ & $47,2 \%$ & $66,7 \%$ & $34,5 \%$ & $48,5 \%$ & $0,0 \%$ \\
Femoral & $0,0 \%$ & $0,0 \%$ & $0,0 \%$ & $0,0 \%$ & $5,1 \%$ & $11,5 \%$ & $25,0 \%$ \\
& & & & & & & \\
Custo Financeiro & $0,3 \%$ & $0,4 \%$ & $0,3 \%$ & $0,3 \%$ & $0,3 \%$ & $1,7 \%$ & $0,5 \%$ \\
\hline
\end{tabular}


Tabela 4 - Distribuição dos pacientes submetidos a acesso vascular para hemodiálise, nas regiões geográficas segundo o tipo de acesso e local anatômico, em números absolutos.

\begin{tabular}{rrrrr}
\hline Variáveis/Regiões & SUL & SUDESTE & NORDESTE & TOTAL \\
\hline FAV & 228 & 1753 & 408 & 2389 \\
Cateter & 7 & 112 & 51 & 170 \\
& & & & \\
Fístula Arteriovenosa & & 1280 & 353 & 1787 \\
Distal & 154 & 400 & 50 & 517 \\
Proximal & 67 & 67 & 4 & 77 \\
Ptfe & 6 & 2 & 0 & 3 \\
Safena & 1 & 4 & 1 & 5 \\
Outras & 0 & 1753 & 408 & 2389 \\
Total & 228 & & & \\
& & 13 & 2 & 15 \\
Cateter & & & & \\
Longa Permanência & 0 & 47 & 24 & 72 \\
Curta Permanência & & 49 & 17 & 72 \\
Jugular & 1 & 3 & 8 & 11 \\
Subclávia & 6 & 99 & 49 & 170 \\
Femoral & 0 & 7 & &
\end{tabular}

Tabela 5 - Distribuição dos pacientes submetidos a acesso vascular para hemodiálise, nas regiões geográficas segundo o tipo de acesso, local anatômico e custo financeiro, em porcentagem.

\begin{tabular}{rrcc}
\hline Variáveis/Regiões & SUL & SUDESTE & NORDESTE \\
\hline FAV & $97,0 \%$ & $94,0 \%$ & $88,9 \%$ \\
Cateter & $3,0 \%$ & $6,0 \%$ & $11,1 \%$ \\
& & & \\
Fístula Arteriovenosa & & & \\
Distal & $67,5 \%$ & $73,0 \%$ & $86,5 \%$ \\
Proximal & $29,4 \%$ & $22,8 \%$ & $12,2 \%$ \\
Ptfe & $2,6 \%$ & $3,8 \%$ & $1,0 \%$ \\
Safena & $0,5 \%$ & $0,1 \%$ & $0,0 \%$ \\
Outras & $0,0 \%$ & $0,3 \%$ & $0,3 \%$ \\
& & & \\
Cateter & & & $4,0 \%$ \\
Longa Permanência & $0,0 \%$ & $11,7 \%$ & $47,0 \%$ \\
Curta Permanência & & & $33,3 \%$ \\
Jugular & $14,2 \%$ & $41,9 \%$ & $15,7 \%$ \\
Subclávia & $85,7 \%$ & $43,7 \%$ & $1,3 \%$ \\
Femoral & $0,0 \%$ & $2,7 \%$ & \\
& & & \\
\hline
\end{tabular}




\section{DISCUSSÃO}

A população estudada foi selecionada em 23 unidades de hemodiálise que recebem orientação da Renal Therapy Service, não apresentando nenhum outro critério de seleção, portanto, os resultados do estudo devem ser interpretados com cautela, pois a amostra avaliada é selecionada e pode não representar adequadamente os serviços brasileiros.

Os cateteres como via de acesso para a hemodiálise, representaram 6,65\% do total da amostra. Esse dado é próximo aos apresentados em várias publicações ${ }^{2,4-7}$ que variou de $5,3 \%$ a 9,3\% e inferiores a outros autores ${ }^{3,8}$ que foram de $19 \%$ e $22,2 \%$.

Hakim e Himmelfarb ${ }^{3}$ publicaram dados que demonstram tendência ao aumento do uso de cateteres como via de acesso para hemodiálise nos últimos anos: $10 \%$ em 1993 e 19\% em 1996. Esses achados vêm ao encontro aos dados apresentados por Tokars et al ${ }^{8}$, que em publicação recente, referente aos anos de 95 , 96, 97 e 99, demonstrou que o uso de cateteres como via de acesso para hemodiálise nos EUA foi de 12,7\%; $14,9 \% ; 17,5 \%$ e $22,2 \%$ respectivamente.

A utilização do cateter como via de acesso para hemodiálise tem aumentado ao longo do tempo e esse fato se deve à preocupação atual em diminuir o uso do PTFE nos EUA. Porém, ao invés de se diminuir o uso do PTFE com o crescimento da utilização das FAV diretas, ocorreu aumento da utilização do cateter de longa permanência como acesso definitivo ${ }^{8}$, o que não corresponde com a finalidade original do uso dos cateteres que foram criados para uso temporário 9.

Rodriguez et al ${ }^{7}$., relataram que a porcentagem do uso de cateter aumenta com a idade dos pacientes, por falência da vasculatura superficial e pela doença arterial periférica e em sua casuística, em pacientes menores de 14 anos nenhum cateter foi verificado, entre 14 e 44 anos a porcentagem encontrada foi de $3 \%$ e acima dos 74 anos, cresceu para $10,2 \%$. No presente estudo, a utilização de cateter como via de acesso para hemodiálise variou, entre as 23 unidades de $0 \%$ a 21,7\%. Entre os estados da União de 1,0\% no Rio Grande do Sul a 11,3\% na Bahia. Entre as regiões geográficas, de 3,0\% na região sul a $11,1 \%$ na região nordeste. Esses dados ficam abaixo dos publicados por Feldman et al ${ }^{9}$ que em estudo realizado em 1372 pacientes que dializavam em 17 centros de hemodiálise de Chicago, encontraram uma variação para o uso de cateteres como via de acesso para hemodiálise de 3,3\% a $45 \%$.
A tendência de se utilizar em menor frequiência o cateter em nosso país quando comparado aos dados americanos, talvez se explique pela grande dificuldade de se aceitar a FAV direta como via principal de acesso vascular para hemodiálise naquele país. No Brasil, a utilização de cateter, na grande maioria das vezes, fica restrita a períodos de espera por um acesso vascular definitivo. A variação encontrada na utilização de cateter em nosso estudo, talvez possa ser explicada pelo fato de haver diferenças estruturais entre as unidades, ou seja, existem unidades que estão localizadas ou ligadas a hospitais de referência regionais e, portanto, recebem maior quantidade de pacientes agudizados o que faz aumentar a utilização de cateteres para hemodiálise, pois esses pacientes chegam à unidade em urgência dialítica e sem via de acesso definitiva. Outras unidades recebem pacientes referendados de ambulatórios específicos de nefrologia e uma grande maioria dos pacientes são encaminhados já com acesso definitivo.

Em relação ao tipo de cateter, de longa permanência ou de curta permanência, o resultado encontrado na casuística do presente trabalho, foi de $8,83 \%$ e $91,17 \%$ respectivamente. Quando comparados aos dados de Rodriguez et al. ${ }^{7}$, que foram de $27,7 \%$ e $72,3 \%$ respectivamente, notamos um maior uso do cateter de longa permanência em seu estudo. Outros autores ${ }^{3,8,9}$ não especificam o tipo de cateter utilizado em suas publicações.

$\mathrm{O}$ uso de cateter de longa permanência, entre as unidades, variou de $0 \%$ a $100 \%$. Entre os estados, a variação foi de $0 \%$ nos estados do Rio Grande do Sul, Paraná e Rio de Janeiro e 25\% no estado de São Paulo. Entre as regiões geográficas a variação foi de $0 \%$ na região Sul e $11,7 \%$ na região sudeste. A inserção dos cateteres de longa permanência, além de ter um custo financeiro maior que o de curta permanência, requer técnica mais apurada em comparação com os cateteres de curta permanência, pois necessita de tunelização do subcutâneo e preferencialmente é inserido na veia jugular interna direita ${ }^{9}$. Esse detalhe técnico requer maior habilidade do profissional e portanto mais tempo de treinamento o que leva a um aumento do custo financeiro do uso deste cateter e talvez seja outro fator importante que possa explicar o pouco uso do referido cateter em nosso país e a alta porcentagem nos estados e regiões mais ricas da União.

Em relação à via de acesso utilizada para a inserção dos cateteres, os resultados da casuística 
foram de $51,1 \% ; 42,4 \%$ e $6,47 \%$ para a inserção na veia jugular interna, veia subclávia e veia femoral respectivamente. Feldman et $_{\text {al. }}{ }^{9}$, relataram que a veia jugular foi a preferencial e não especificam a porcentagem do uso dessa via. Hakim e Himmelfarb3, exaltam a diminuição do uso da veia subclávia como via de acesso de 21,6\% em 1993 para 9\% em 1996. Rodriguez et al. ${ }^{7}$, detalham melhor os locais anatômicos utilizados para a inserção dos cateteres e encontraram: $75,1 \%, 22,0 \%$ e $2,9 \%$ para as veias jugular interna, subclávia e femoral respectivamente, sendo o lado direito escolhido na grande maioria das vezes, não referindo porcentagem. Entre os estados da União, a utilização da veia subclávia como via de acesso para inserção de cateter, variou de $0 \%$ a $100 \%$. Entre as regiões geográficas, a utilização da veia subclávia variou de $33,3 \%$ na região Nordeste a $85,7 \%$ na região Sul. Podemos notar que a utilização da veia subclávia como via de acesso para inserção de cateter em nosso país é superior aos dados da literatura acima descritos.

A preocupação atual com a estenose da veia subclávia devido ao uso dessa veia como via de acesso para a inserção de cateter, é descrita em vários artigos da literatura ${ }^{3,10}$. A técnica de punção e inserção do cateter em veia subclávia é mais popular em nosso país, talvez por ser de domínio da grande maioria dos médicos, pois aprendemos nas escolas que a veia subclávia é uma excelente opção como via de acesso central. Portanto, os médicos são mais treinados em punção da veia subclávia do que da veia jugular. Por ter mais habilidade com a veia subclávia, a utilização da veia subclávia tem a preferência em nosso meio. Somente com programas de treinamento e conscientização dos médicos, poderemos diminuir a utilização dessa via de acesso.

Do total de 2559 pacientes estudados, 2389 tinham acesso vascular direto através de FAV o que corresponde a 93,4\%. Dessa população, constatouse que $96,5 \%$ das FAV eram diretas; $3,2 \%$ com a interposição de PTFE; 0,1\% eram construídas com interposição de segmento de veia safena e $0,2 \%$ foram consideradas como outras. Esses dados, quando comparados aos da literatura, mostram proximidade apenas aos dados de $\mathrm{Sesso}^{2}$ que relata o uso do FAV com prótese em $2 \%$. Em relação à literatura internacional, o uso da prótese em nosso meio é inferior aos encontrados por Rodriguez ${ }^{7}$ e Besarab $^{11} \mathrm{e}$ muito inferior aos dados encontrados na literatura americana que chegam a atingir a $85 \%^{12}$.
A variação do uso de PTFE entre as unidades foi de $0 \%$ a $17 \%$. Entre os estados da união, a variação foi de $0,8 \%$ nos estados do Paraná e Bahia a 5,0\% no Rio Grande do Sul. Entre as regiões geográficas essa variação foi de $1,0 \%$ na região Nordeste a $3,8 \%$ na região Sudeste. Essa variação é também inferior à apresentada por Hirth et al. ${ }^{12}$ que mostram uma variação do uso de prótese de $23 \%$ a $85 \%$.

O uso abusivo do PTFE nos EUA é a grande preocupação da maioria dos autores daquele país, que na última década tem apresentado publicações demostrando o uso abusivo da prótese e como conscientizar os profissionais envolvidos com o acesso para hemodiálise que esse uso é a principal causa de morbidade, mortalidade, internações e aumento do custo com a hemodiálise ${ }^{3,5,9,11-25}$.

No Brasil, o uso do PTFE é muito inferior aos apresentados na literatura e talvez possamos ser um grande exemplo a ser seguido por outros países. O baixo uso do PTFE em nosso meio talvez possa ser explicado pelo fato do custo financeiro da prótese ser alto. Como $96 \%$ do pagamento da hemodiálise no Brasil é feito pelo Ministério da $\mathrm{Saúde}^{2}$, e a verba destinada é pequena, a possibilidade de se manter a prótese em estoque nos nossos hospitais, é menor que nos países desenvolvidos. Besarab ${ }^{11}$ descreve que outro fator importante para o "sucesso" do PTFE nos EUA é que $50 \%$ das enfermeiras e $25 \%$ dos nefrologistas preferem a prótese e portanto o "feed back" que os cirurgiões têm desses profissionais é a favor da prótese. As enfermeiras preferem a prótese pela facilidade de punção, e os nefrologistas pelo menor tempo de utilização que esse tipo de acesso necessita para poder ser utilizado, quando comparado às FAV diretas que em média necessitam de um tempo que varia de três a seis semanas. Em relação aos cirurgiões, o autor comenta que a preferência pela prótese, além do "feed back" da equipe técnica e dos nefrologistas, pode existir uma suspeita de considerações financeiras, pois os honorários médicos são maiores quando se coloca uma prótese.

Os estados brasileiros com maior taxa de PTFE foram os estados do Rio Grande do Sul, São Paulo, Minas Gerais e Rio de Janeiro com 5,0\%; 4,4\%; $4,3 \%$ e $2,5 \%$ respectivamente. Esses dados sugerem que o uso do PTFE está mais concentrado nos estados mais desenvolvidos da União, o que corrobora com as colocações acima descritas.

Da totalidade de 2389 acessos construídos, constatamos a utilização da veia safena interna 
interposta como substituto no membro superior em três pacientes $(0,1 \%)$ e as FAV consideradas como outras em cinco $(0,2 \%)$ assim distribuídas: artéria tibial posterior e a veia safena na região maleolar em três pacientes e artéria poplítea e a veia safena na altura do joelho em dois. Em publicações mais recente, o uso da veia safena para construção de acesso vascular foi relatada por Bender et al. ${ }^{13}$ que em 104 operações, a utilizaram em cinco oportunidades $(4,8 \%)$. Rodriguez et al. $^{7}$ relataram o uso da veia safena em 5,7\% sendo que desta totalidade, $53 \%$ foram realizadas no membro inferior e $47 \%$ no membro superior. Albers ${ }^{26}$ relatou que a preferência pelo PTFE sobre a veia safena ocorre pela facilidade de obtenção da prótese e pela necessidade de se preservar a veia safena para possíveis operações de revascularização cardíacas ou de membros inferiores que possam vir a ocorrer.

A literatura atual é relativamente pobre no que diz respeito a criação de FAV consideradas como outras, realizadas na grande maioria nos membros inferiores. Huber et $a l^{23}$, relataram o uso da veia femoral superficial transposta para o membro superior em dois casos. Polo et al. ${ }^{18}$, publicaram artigo sobre a criação de uma FAV com a interposição de um pequeno segmento de PTFE entre a artéria braquial e a veia cefálica em 222 operações e concluíram que a técnica apresentou um bom resultado com uma taxa de complicação de 0,22/ano, menor que a encontrada com a FAV construída com PTFE que foi de 0,49/ano para o mesmo período.

Os acessos vasculares considerados como outros, ficaram restritos a situações em que a possibilidade de se criar um acesso vascular já estava esgotada e, portanto, são operações consideradas como última opção e só são realizadas quando realmente todas as outras alternativas não são mais possíveis, $o$ que explica o seu pouco uso.

Em relação à localização das FAV diretas, as distais foram realizadas em $74,8 \%$ e as proximais em $21,7 \%$. Em nosso meio, a preferência pelas FAV distais foi relatada em vários estudos. ${ }^{26-28}$ Quando comparado aos dados da literatura internacional atual, notamos que a utilização das FAV distais em nosso meio é mais aceita que a grande maioria das publicações. Rodriguez et al. ${ }^{7}$ apresentam como resultados, $66,3 \%$ para as FAV distais e $33,7 \%$ para as proximais. Ascher et al. ${ }^{22}$, apresentaram dados em que as FAV distais foram construídas em $47 \%$ e as proximais em $52 \%$ sendo $30 \%$ braquiocefálica e $22 \%$ braquiobasílica
Sands ${ }^{19}$, em publicação recente, apresentou um programa de melhoria da qualidade do acesso vascular e demonstrou o crescimento do uso da artéria braquial para confecção de FAV nos anos de 1993, 1994 e 1995 comparado ao período de 1990 a 1992, que foi de $16,1 \%$ para $43,7 \%$.

Em 1990, Ryan e Dennis ${ }^{29}$ descreveram a FAV distal realizada no punho com a artéria radial e a veia cefálica como a FAV ideal. Usou como argumentos principais: o trajeto longo e superficial da veia cefálica, permitindo assim vários pontos possíveis de punção, a preservação da vasculatura acima do punho que permitiria, caso necessário, a realização de nova FAV utilizando o mesmo membro, por ser a FAV que apresenta menor número de complicações ao longo do tempo e que apresenta uma boa longevidade de sua função. Como contra argumentos podemos citar a sua alta porcentagem de falha primária que pode variar de $9 \%$ a $30 \%{ }^{9}$ e a necessidade de um alto fluxo sanguiíneo na hemodiálise de alta eficiência, o que teoricamente a FAV distal não seria capaz de suprir, principalmente em pacientes do sexo feminino ${ }^{14}$.

As FAV proximais são consideradas como os acessos de segunda opção, dado esse sugerido no NKF-DOQI ${ }^{30}$ que coloca como primeira opção a FAV radiocefálica, como segunda opção a FAV braquiocefálica e discute a terceira opção entre a FAV braquiobasílica superficializada e a FAV com a interposição do PTFE. Ascher et al. ${ }^{22}$, em seu protocolo de escolha colocaram a FAV rádiocefálica como primeira opção, a FAV braquiocefálica como segunda e a FAV braquiobasílica superficializada como terceira, deixando a utilização do PTFE como quarta.

Em nosso meio, os estudos com as FAV distais e proximais estão bem documentados ${ }^{25-27}$. Nas publicações, os autores concluíram que as FAV proximais se mostram técnicas válidas para a manutenção de pacientes com insuficiência renal crônica terminal em hemodiálise, principalmente quando a possibilidade de se construir uma FAV na região do punho não for mais possível.

Os custos financeiros com o acesso vascular para hemodiálise nas 23 unidades representou em média $1,0 \%$ do custo total das unidades com uma variação de $0,3 \%$ a $2,6 \%$. As diferenças entre os custos pode estar relacionada à utilização de cateter como via de acesso para hemodiálise, pois se observarmos a porcentagem do uso de cateter no estado do Rio Grande do Sul que foi de 1,0\% e no estado da Bahia que foi de $11,3 \%$ e relacionarmos com os custos desses 
estados que foram de $0,3 \%$ e $1,7 \%$ respectivamente, podemos suspeitar que a diferença entre os custos está diretamente relacionada com a utilização do cateter. Quando comparados aos dados americanos, que chegam a atingir $25 \%$ do custo totaP, podemos notar que a realidade brasileira em relação ao acesso vascular é muito diferente da realidade americana. A quase totalidade da literatura americana está baseada em diminuição dos custos com acesso vascular que tem como principal responsável a alta incidência do uso do PTFE e dos cateteres.

O programa de hemodiálise é o de maior alcance para o tratamento dos doentes portadores de insuficiência renal crônica terminal. No Brasil, em 1983 havia 2552 pacientes em programa de hemodiálise ${ }^{31}$. Em março de 2000, os dados do Data SUS ${ }^{1}$ contam mais de 40000 pacientes em hemodiálise o que correspondem a 268 pmp. Nos EUA mais de 300000 pacientes estavam em programa de diálise correspondem a 1105 ppm. ${ }^{32}$; no Reino Unido 13466 pacientes com 539 ppm $^{33}$ e 19424 no Canadá ${ }^{6}$. Esses dados traduzem a importância da atividade cirúrgica para a construção de acessos vasculares para hemodiálise assim como a sua manutenção.

A importância do acesso vascular para hemodiálise tem sido reconhecida ultimamente nos
EUA, talvez pelo alto custo, que pode atingir cifras de um bilhão de dólares ao ano ${ }^{19} \mathrm{e}$ levou os autores americanos a se aprofundarem nos conhecimentos do acesso vascular e criarem programas de melhoria de qualidade. $\mathrm{O}$ alto custo com os acessos vasculares nos EUA está diretamente relacionado com o uso abusivo do PTFE, sua construção e principalmente sua manutenção que necessita de duas a três vezes mais procedimentos secundários que a FAV direta, utilizando métodos diagnósticos para prevenir a trombose, diagnosticando com antecedência as possíveis estenoses e tratando-as, ou através da angiorradiologia intervencionista, ou por meio de intervenções cirúrgicas convencionais. Essa tendência, além de elevar os custos financeiros do acesso vascular, aumenta a morbidade, a mortalidade e o sofrimento dos pacientes renais crônicos.

A literatura nacional em relação ao acesso vascular é pobre e pelos dados acima apresentados, compreende-se a importância de se conhecer os dados relativos aos acessos vasculares para hemodiálise em nosso país e demonstrar que a realidade nacional tem suas próprias características. Quando um cirurgião necessita recorrer a uma revisão bibliográfica, fica à mercê da literatura internacional e portanto diferente da nossa realidade.

\begin{abstract}
Background: To demonstrate type and local of vascular access for hemodialysis used in 23 dialysis centers in seven Brazilian states. Methods: From October 1999 to August 2000, a total of 2559 patients in 23 hemodialysis centers were studied to determine: $A$ - the frequency of catheter utilization and arteriovenous fistulae (AVF) as a vascular access for hemodialysis .B-the types of catheters (cuffed, tunneled catheters) or acute one and the sites of choice for the insertion. $C$-if AVF was the access, its type (native or with insertion of polytetrafluoroethylene-PTFE) and location (distal or proximal). D- the costs with vascular access as a percentage of the total costs of the unit. Results: The study showed that $93.4 \%$ of the patients had an AVF as a vascular access for hemodialysis and the remaining $6.6 \%$ had a catheter. Distal native AVF was the more frequently constructed (74.8\%), while the proximal native AVF was constructed in $21.7 \%$ of the times. Insertion of PTFE reached $3.2 \%$, saphenous vein was used in $0.1 \%$ and the others AVFs, $0.2 \%$. Cuffed, tunneled catheters (long term catheters) were used in $8.7 \%$ and acute catheters in $91.3 \%$. The sites of choice when using an acute catheter were the subclavian and internal jugular veins (both with $42.4 \%$ of the preference) and the femoral vein in $6.5 \%$. The costs with a vascular access for hemodialysis reached $1 \%$ of the total costs of the unit. Conclusions: Vascular access for hemodialysis in Brazil has different aspects compared with United States of America or European data. The most frequently vascular access constructed is the distal native AVF and the use of PTFE for AVF construction is very low.
\end{abstract}

Key words: Renal dialysis; Hemodialysis units, hospital; Arteriovenous fistula; Catheters indwelling; Catheter; Kidney failure, chronic 


\section{REFERÊNCIAS}

1 - IDB-2000 Indicadores e dados básicos para a saúde Brasil. [Capturado em 11 Set 2001]; Disponível em: URL: http://www.datasus.gov.br.

2 - Sesso R. Inquérito epidemiológico em unidades de diálise do Brasil. J Bras Nefrol, 2000, 22(3, Supl 2): S2326.

3 - Hakim R, Himmelfarb J - Hemodialysis access failure: a call to action. Kidney Int, 1998, 54(4):1029-1040.

4 - USRDS United States Renal Data System 1995 Annual Data Report. The cost effectiveness of alternative types of vascular access and the economic cost of ESRD. Am J Kidney Dis, 1995, 26(4, Supl 2): S140156.

5 - Sands J, Miranda CL - Increasing numbers of AV fistulas for hemodialysis access. Clin Nephrol, 1997, 48(2): 114-117.

6 - Canadian Institute for Health Information. Canadian Organ Replacement Register. Annual Report 1998. Dialysis and renal transplantation. Ottawa, The Institute, 1998.

7 - Rodríguez JA, López J, Clèries M, et al. - Vascular access for haemodialysis: an epidemiological study of the Catalan Renal Registry. Nephrol Dial Transplant, 1999, 14(7): 1651-1657.

8 - Tokars JI, Miller ER, Alter MJ, Arduino MJ. National Surveillance of Dialysis-Associated Diseases in the United States, 1999. Atlanta, National Center for Infectious Diseases, 2001.

9 - Feldman HI, Kobrin S, Wasserstein A - Hemodialysis vascular access morbidity. J Am Soc Nephrol, 1996, 7(4): 523-535.

10 - Schwab SJ, Quarles LD, Middleton JP, et al. - Hemodialysis-associated subclavian vein stenosis. Kidney Int, 1988, 33(6): 1156-1159.

11 - Besarab A - Why not us? Increasing autologous AV fistulae. In American Society of Nephrology Postgraduate Course. Expanding our procedural domain vascular access, cathethers and ultrasonography. Florida, 1999. (Renal Week 1999, november 3-4, 1999)

12 - Hirth RA, Turenne MN, Woods JD, et al. - Predictors of type of vascular access in hemodialysis patients. JAMA, 1996, 276(16): 1303-1308.

13 - Bender MH, Bruyninckx CM, Gerlag PG - The brachiocephalic elbow fistula: a useful alternative angioaccess for permanent hemodialysis. J Vasc Surg, 1994, 20(5): 808-813.

14 - Kaufman JL - The decline of the autogenous hemodialysis access site. Semin Dial, 1995, 8: 59-61.

15 - Allon M, Bailey R, Ballard R, et al. - A multidisciplinary approach to hemodialysis access: prospective evaluation. Kidney Int, 1998, 53(2): 473-479.
16 - Beathard GA. Vascular access evaluation: the physical examination. In American Society of Nephrology Postgraduate Course. Expanding our procedural domain vascular access, cathethers and ultrasonography. Florida, 1999. (Renal Week 1999, november 3-4, 1999)

17 - Besarab A. Introduction: overview of vascular access. In American Society of Nephrology Postgraduate Course. Expanding our procedural domain vascular access, cathethers and ultrasonography. Florida, 1999. (Renal Week 1999, november 3-4, 1999)

18 - Polo JR, Vázquez R, Polo J, et al. - Brachiocephalic jump graft fistula: an altenative for dialysis use of elbow crease veins. Am J Kidney Dis, 1999, 33(5): 904909.

19 - Sands JJ - Vascular access: how to increase the number of autologous A/V fistulas. In American Society of Nephrology Postgraduate Course. Expanding our procedural domain vascular access, cathethers and ultrasonography. Florida, 1999. (Renal Week 1999, november 3-4, 1999)

20 - Konner K - A primer on the av fistula-Achilles' heel, but also Cinderella of haemodialysis. Nephrol Dial Transplant, 1999, 14(9): 2094-2098.

21 - Besarab A, Escobar F - A glimmer of hope: increasing the construction and maturation of autologous arteriovenous fistulas. Am J Kidney Dis, 1999, 33(5): 977979.

22 - Ascher E, Gade P, Hingorani A, et al. - Changes in the practice of angioaccess surgery: impact of dialysis outcome and quality initiative recommendations. J Vasc Surg, 2000, 31(1 pt 1): 84-92.

23 - Huber TS, Ozaki CK, Flynn TC, et al. - Use of superficial femoral vein for hemodialysis arteriovenous access. J Vasc Surg, 2000, 31(5): 1038-1041.

24 - McMurray SD, Miller J - Using CQI to improve vascular access management in the dialysis unit. Nephrol News Issues, 2000, 14(12): 25-29.

25 - Marx AB, Landmann J, Harder FH - Surgery for vascular access. Curr Probl Surg, 1990, 27(1):1-48.

26 - Albers MTV. Análise crítica das fístulas arteriovenosas para acesso vascular à hemodiálise. Dissertação (Mestrado). São Paulo. Universidade de São Paulo, 1985.

27 - Linardi F. Fístula arteriovenosa para hemodiálise: estudo comparativo entre as fístulas proximais e distais. Dissertação (Mestrado). Sorocaba. Pontífice Universidade Católica, 1993.

28 - Frankini AD. Fístulas arteriovenosas braquiocefálicas e braquiobasílicas para hemodiálise nos seguimentos imediato, precoce e tardio. Dissertação (Mestrado). São Paulo, Escola Paulista de Medicina, 1994. 
29 - Ryan JJ, Dennis MJ - Radiocephalic fistula in vascular access. Br J Surg, 1990, 77(12): 1321-1322.

30 - NKF-DOQI Clinical practice guidelines for vascular acess. National Kidney Foundation. Dialysis Outcomes Quality Initiative. Am J Kidney Dis, 1997, 30 (4 Suppl 3): 150-189.

31 Registro brasileiro de diálise e transplante. J Bras Nefrol, 1984, 6(3): 89-96.

32 USRDS United States Renal Data System 1999 Annual Data Report. April 1999. [Capturado em 29 Ago 2001]; Disponível em: URL: http://www.usrds.org.
33 - Ansell D, Feest T, Will E, Burden R, Smith S, Lewis M, et al. The Second Annual Report. The UK Renal Registry December 1999 [Capturado em 29 Ago 2001]; Disponível em: URL: http://www.renalreg.com.

Endereço para correspondência:

Dr. Fábio Linardi

Av. São Paulo, 2.918

Jd. Paulista - Sorocaba - SP

CEP: 18031-004 